\title{
Corela
}

Cognition, représentation, langage

$12-2 \mid 2014$

Vol. $12, n^{\circ} 2$

\section{Expression de l'exclamation en anglais au moyen de marqueurs atypiques}

\section{Bénédicte Guillaume et Emmanuel Baumer}

\section{(2) OpenEdition}

\section{Journals}

Édition électronique

URL : http://journals.openedition.org/corela/3654

DOI : $10.4000 /$ corela.3654

ISSN : $1638-573 \mathrm{X}$

\section{Éditeur}

Cercle linguistique du Centre et de I'Ouest - CerLICO

\section{Référence électronique}

Bénédicte Guillaume et Emmanuel Baumer, «Expression de l'exclamation en anglais au moyen de marqueurs atypiques », Corela [En ligne], 12-2 | 2014, mis en ligne le 15 décembre 2014, consulté le 01 mai 2019. URL : http://journals.openedition.org/corela/3654; DOI : 10.4000/corela.3654

Ce document a été généré automatiquement le 1 mai 2019.

\section{(c) (i) (2)(2)}

Corela - cognition, représentation, langage est mis à disposition selon les termes de la licence Creative Commons Attribution - Pas d'Utilisation Commerciale - Partage dans les Mêmes Conditions 4.0 International. 


\title{
Expression de l'exclamation en anglais au moyen de marqueurs atypiques
}

\author{
Bénédicte Guillaume et Emmanuel Baumer
}

\begin{abstract}
Nous remercions nos collègues de l'équipe "Linguistique de l'énonciation " (dirigée par G. Salvan) de l'UMR 7320 Bases, Corpus, Langage (Univ. Nice Sophia Antipolis et CNRS) pour leurs remarques sur une version antérieure de ce travail, présentée dans le cadre du programme "Approche énonciative et pragmatique de l'exclamation", dont le responsable est $R$. Faure. Nous avons également pu bénéficier de retours de la part des participants à la journée d'étude " Marqueurs d'intensité et positionnement énonciatif » organisée le 27 juin 2014 à l'Université de Paris Est Marne la Vallée par H. Bays et L. Dufaye, lors de laquelle nous avons communiqué sur le présent sujet. Nous restons néanmoins seuls responsables des erreurs ou inexactitudes pouvant figurer dans cet article.
\end{abstract}

\section{Introduction}

1 Si la plupart des définitions du contexte exclamatif sont centrées sur l'énonciateur et ses attentes ${ }^{1}$, nous essaierons de montrer à travers nos exemples que la prise en compte de la relation intersubjective est tout aussi indispensable que celle des attentes de l'énonciateur. A cette fin, nous considérons l'exclamation comme une catégorie grammaticale à part entière ${ }^{2}$, celle de l'hyper assertion ${ }^{3}$. Une telle approche permet également de différencier l'exclamation de certains procédés expressifs ${ }^{4}$, tels la mise en relief, l'emphase, la proéminence, qui, pour leur part, désignent des phénomènes marquant un haut degré d'intensité auxquels on a fréquemment recours en contexte exclamatif, sans pour autant que leur présence à elle seule soit suffisante pour déclencher de manière systématique une interprétation exclamative.

Dans le présent travail, nous nous intéressons à des manières atypiques d'exprimer l'exclamation en anglais, et plus particulièrement à deux phénomènes distincts : les énoncés comportant un question tag (ci-après QTag) d'une part, et certains énoncés comportant le marqueur some d'autre part. Nous considérons ces phénomènes comme 
non typiques de l'exclamation, d'une part parce qu'ils ne sont pas emblématiques de cette catégorie grammaticale, et d'autre part parce que seule une minorité d'exemples de ce type est concernée par une interprétation exclamative.

3 Or, si certains exemples de ces catégories peuvent malgré tout être analysés comme exclamatifs, c'est avant tout en raison d'une relative compatibilité au niveau des marqueurs employés : marqueurs syntaxiques (inversion sujet / auxiliaire systématique dans les QTags, possibilité de la présence d'un point d'exclamation pour certains énoncés comportant some...), et surtout marqueurs prosodiques ${ }^{5}$. On trouve ainsi dans nos exemples une amplification et une intensité normalement attendues dans les énoncés exclamatifs : grandes chutes, grandes montées, amplitude, allongement des voyelles ${ }^{6} . .$.

Or, de tels marqueurs syntaxiques et prosodiques ne sont après tout pas exclusifs d'une interprétation exclamative, et ils ne sont pas non plus indispensables pour que celle-ci intervienne. Dès lors, ce qui permet d'attribuer une interprétation exclamative à certains emplois de QTags ou à certains énoncés avec some, est avant tout la prise en compte du contexte. Ainsi, l'interprétation exclamative de nos exemples correspond en général à un contexte dans lequel on constate un haut degré de différenciation entre les positionnements respectifs de l'énonciateur et du co-énonciateur. Une telle altérité signale un contexte polémique, qui est un bon déclencheur de l'hyper assertion, typique pour sa part d'une interprétation exclamative. Il $\mathrm{y}$ a donc bel et bien une mise à mal des attentes de l'énonciateur, mais celle-ci est à mettre au compte des propos et / ou de l'attitude du co-énonciateur, qui ne se conforme pas à ce que l'énonciateur avait espéré ou supposé. Dans la mesure où la prise en compte de la dimension intersubjective dans l'interprétation exclamative nous semble essentielle, nous avons recours dans notre étude aux outils de la Théorie des Opérations Enonciatives d'A. Culioli, un cadre théorique qui donne toute sa place à la relation intersubjective.

\section{Les énoncés avec QTag exclamatifs}

5 Il peut sembler paradoxal d'attribuer à certains énoncés avec QTag une fonction exclamative, puisque cette catégorie est plus facilement assimilée à celle de l'interrogation, en raison de la présence systématique d'un point d'interrogation en fin d'énoncé dans les exemples qui vont suivre. Or, nous expliquons par ailleurs (Guillaume 2006) qu'il est nécessaire de remettre en cause la caractérisation des QTags comme étant des questions (tronquées) ou bien des demandes de confirmation. Il existe en réalité plusieurs catégories d'énoncés avec QTag, distinctes tant dans leur forme (type de phrase dans la base ${ }^{7}$, inversion de polarité ou pas, auxiliaires employés, intonation...) que dans leur contexte privilégié d'emploi. Néanmoins, il est possible de proposer un invariant pour l'ensemble des énoncés avec QTag de l'anglais, à savoir le fait que l'énonciateur postule un repérage du contenu de la base en fonction du co-énonciateur, ce qui est partie prenante d'une stratégie énonciative.

6 Ainsi, lorsque l'énonciateur emploie un QTag, il attribue ce faisant un positionnement au co-énonciateur vis-à-vis de la prise en charge du contenu de la base. Or, la plupart du temps, il y a bien attribution, et non demande, de prise en charge, ce qui signifie que l'énonciateur ne cherche pas vraiment à connaître l'opinion du co-énonciateur, d'où l'impossibilité de caractériser l'ensemble des QTags comme étant des questions ou des demandes de confirmation: certains le sont, mais c'est en fait une minorité. Ainsi, la catégorie d'énoncés avec QTag la plus courante est celle permettant d'asserter sans 
réserve le contenu de la base tout en le repérant en fonction du co-énonciateur : on trouve typiquement dans cette catégorie des dictons, des lieux communs (par exemple: It's a small world, isn't it?). Les positionnements des co-énonciateurs sont alors identifiés : «ce que je dis, tu pourrais le dire aussi ».

(1) Sculptress-11.51.052 :

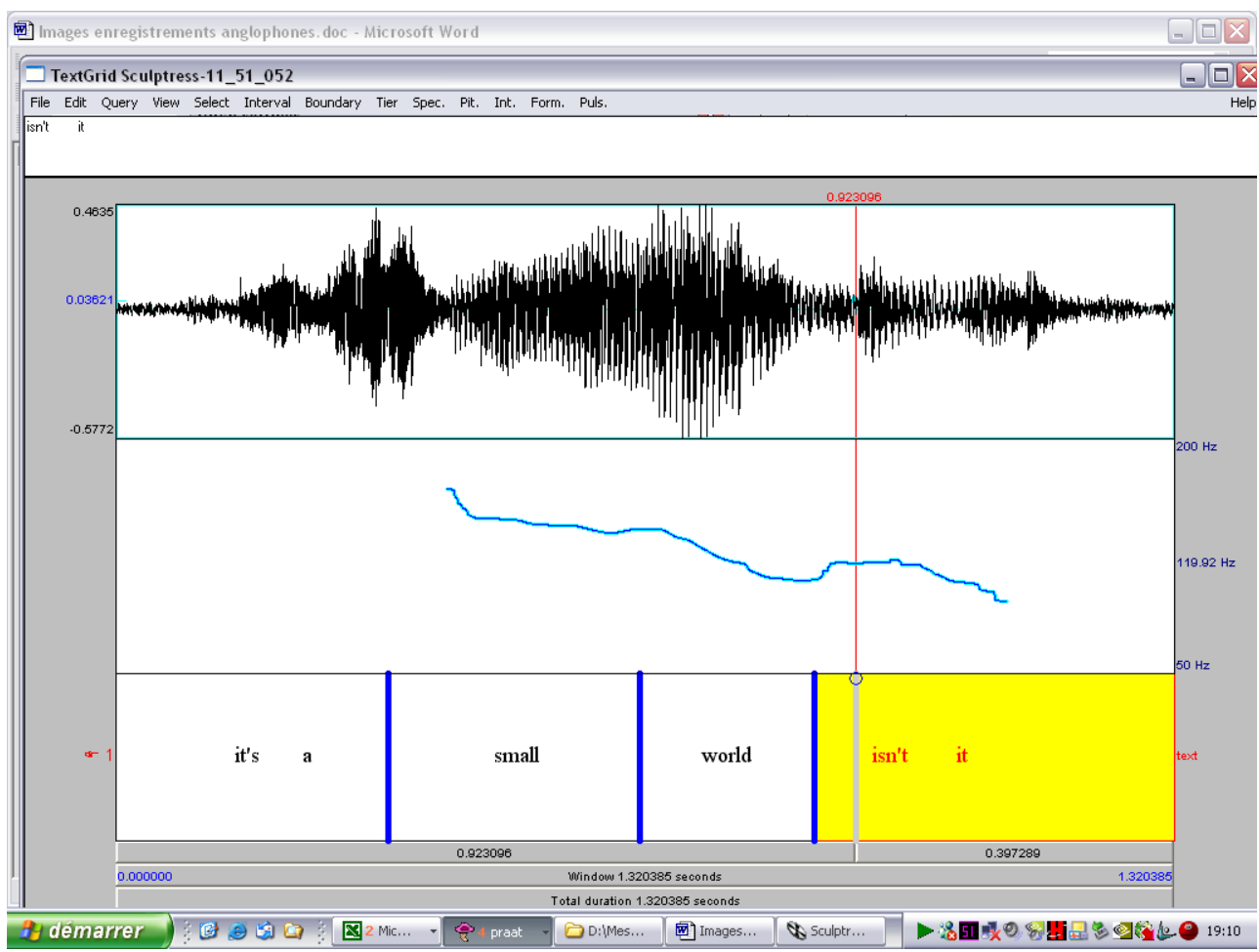

On remarque dans l'enregistrement de cet exemple (extrait d'une série britannique) la faible amplitude intonative (approximativement entre 100 et $150 \mathrm{hz}$ ), qui s'explique par une absence de polémique, une absence d'altérité : la position de l'énonciateur et celle du co-énonciateur sont présentées comme identifiées, sans qu'aucun élément du contexte ne semble aller à l'encontre de cette supposition. Ainsi, les énoncés avec QTag de cette catégorie ne peuvent jamais être compatibles avec l'expression de l'exclamation. Tournons-nous de ce fait vers une autre catégorie d'énoncés avec QTag, qui se révèle nettement plus compatible avec le thème abordé ici :

(2) A few days before she had drawn his horoscope and this morning she had told him the single key number of his name was eight. (...)

'You eight people,' she said, 'often appear cold and undemonstrative with those you ought to love and trust.'

'Cold?' he said. 'Undemonstrative? You must be joking. You are joking, aren't you, Senta?

'It's because you're afraid of being considered weak. (...)' (Bridesmaid 93)

« Dis-moi que tu plaisantes $!^{9}$ »

En utilisant un QTag, Philip, le jeune homme qui parle, fait comme si Senta était forcément d'accord avec lui, mais le contexte démontre qu'il prend en réalité l'exact contre-pied de ce que l'attitude de la jeune femme laisse supposer : elle n'a en effet pas du tout l'air de plaisanter et prend visiblement très au sérieux cette histoire de numérologie. 
Dans un tel cas de figure, l'emploi d'un QTag vient donc s'opposer à un préconstruit contraire attribué au co-énonciateur. Il y a dès lors exacerbation de la différenciation entre les positionnements respectifs des co-énonciateurs, car, contre toute évidence, l'énonciateur postule que le co-énonciateur a une position identique à la sienne, dans le

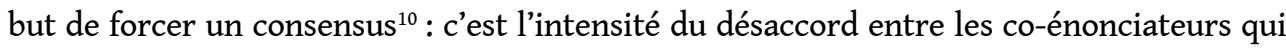
donne lieu ici à un effet de sens exclamatif pour l'ensemble de l'énoncé avec QTag, puisque, comme nous l'avons expliqué en introduction, un contexte polémique déclenche presque toujours une interprétation exclamative en ce qui concerne les phénomènes qui nous intéressent ici.

Bien que la présence d'un point d'interrogation ne soit a priori guère compatible avec l'exclamation, il faut admettre que cette marque de ponctuation est en réalité vide de sens dans un tel QTag. Ainsi, nous proposons une traduction en français qui nous semble reprendre l'esprit de l'énoncé anglais original sinon sa forme, et qui a recours pour sa part à un point d'exclamation (cf. supra).

11 Avec un tel exemple, on se trouve très proche de la définition du contexte exclamatif donnée en introduction : le préconstruit contraire, c'est-à-dire l'attitude et les paroles du co-énonciateur, met en défaut les attentes de l'énonciateur, et l'oblige dès lors à hyper asserter la description de ce qui correspond à ses attentes. L'emploi du QTag comme de l'appellatif s'interprètent comme un effort un peu désespéré de changer la réalité, en forçant sur elle une interprétation qui de manière évidente n'est pas la bonne («c'est une plaisanterie!»). Or, ce jeu autour de la présence d'un préconstruit contraire, et la manière dont l'énonciateur choisit de s'y opposer, presque de toutes ses forces, est un contexte hautement compatible avec l'expression de l'exclamation :

On a en effet l'idée que, pour qu'il y ait exclamation, il faut qu'il y ait un préconstruit. Mais en fait il y a une rupture par rapport à ce préconstruit dans le cas de la surprise. Par conséquent on trouve à la fois l'existence du préconstruit et quelque chose qui s'y oppose de façon violente, qui le forclot. (Danon-Boileau 1995 : 244 ; c'est nous qui mettons en gras)

12 En ce qui concerne l'intonation de l'exemple (2), on rencontre dans l'enregistrement sollicité que nous en avons réalisé une proéminence intonative qui atteint environ 200 $\mathrm{Hz}$. Cette dernière précède une grande chute en ce qui concerne l'auxiliaire de la base, tandis que l'auxiliaire du QTag reprend cette caractéristique a minori (cf. Gaudy-Campbell 2002), avec une chute qui débute à environ $150 \mathrm{~Hz}$. On remarque aussi que l'auxiliaire de la base comme celui du QTag sont prononcés avec une intensité marquée, ce qui est là aussi un trait exclamatif assez typique, et est dû en l'occurrence à la présence en contexte d'un préconstruit contraire : 


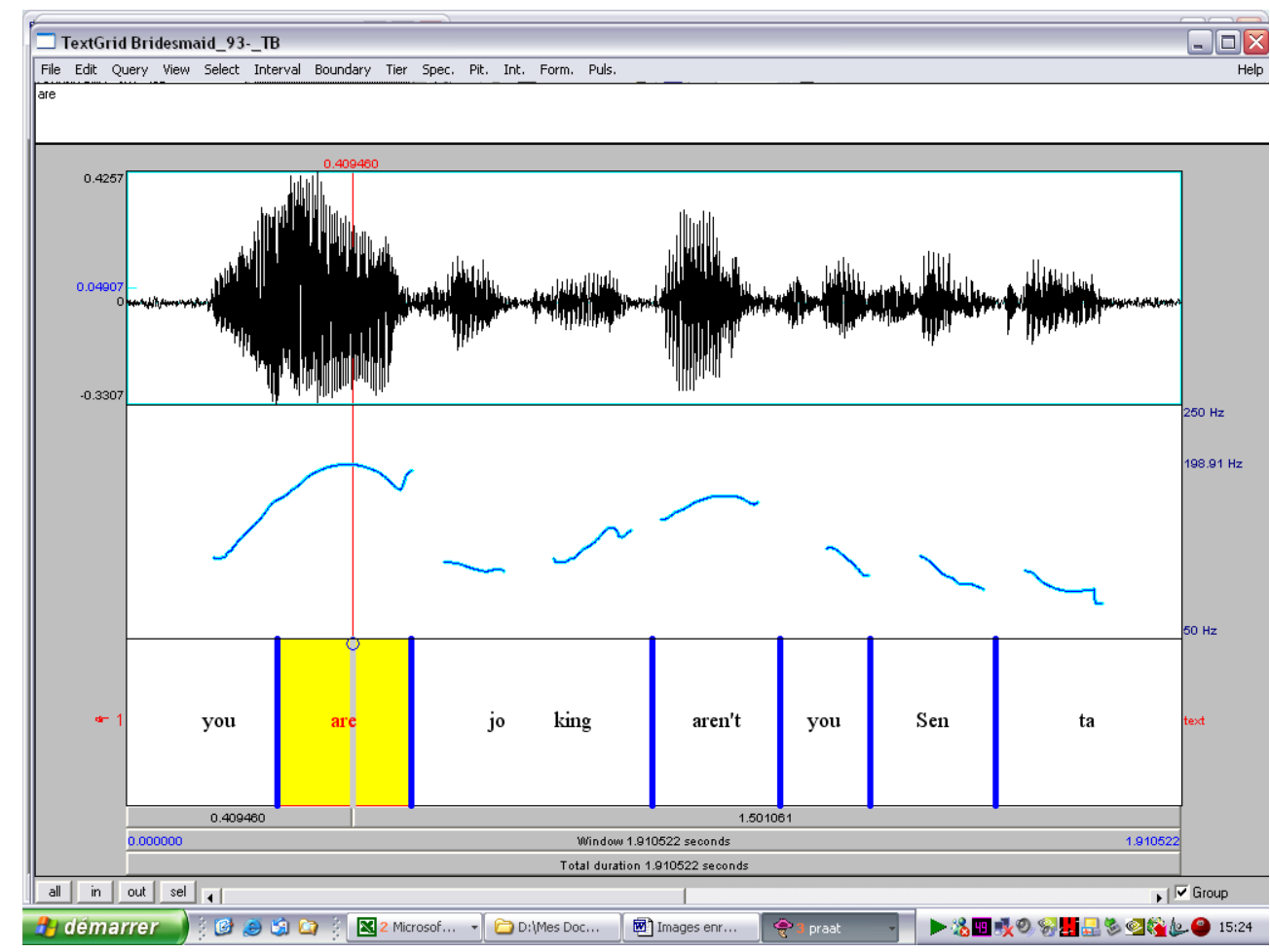

Outre la présence d'un préconstruit contraire, d'une hyper assertion et de caractéristiques intonatives compatibles avec l'expression de l'exclamation, la présence d'une inversion sujet / auxiliaire au niveau du QTag est un autre élément facilitant une telle interprétation. En effet, une inversion sujet / auxiliaire empêche la pleine validation de la valeur positive ou bien de la valeur négative pour une relation prédicative donnée, car elle engendre un parcours ${ }^{11}$. Dans un contexte exclamatif, ce parcours se stabilise par une sortie vers le haut du domaine notionnel (cf. Culioli 1999): c'est le haut degré1 ${ }^{12}$, comme dans l'exemple suivant emprunté à J. Rett : (Boy,) Does John bake delicious desserts! (2011: 412).

En ce qui concerne le contexte de l'exemple suivant, Fran Boyce accuse son compagnon, un homme du nom de McCullough, de violences conjugales. L'avocate de Fran est en train d'interroger McCullough. Celui-ci reconnaît s'être disputé avec son amie, mais nie les coups. Le rôle de l'avocate est donc de les lui faire avouer devant le juge afin qu'il puisse être condamné ${ }^{13}$. Après l'avoir amené à raconter le début de la dispute, elle enchaîne comme suit :

(3) Avocate de Fran Boyce: And you struck her, did you not? McCullough : No, I didn't hit her. (Corner-QTII-35.27.202) 


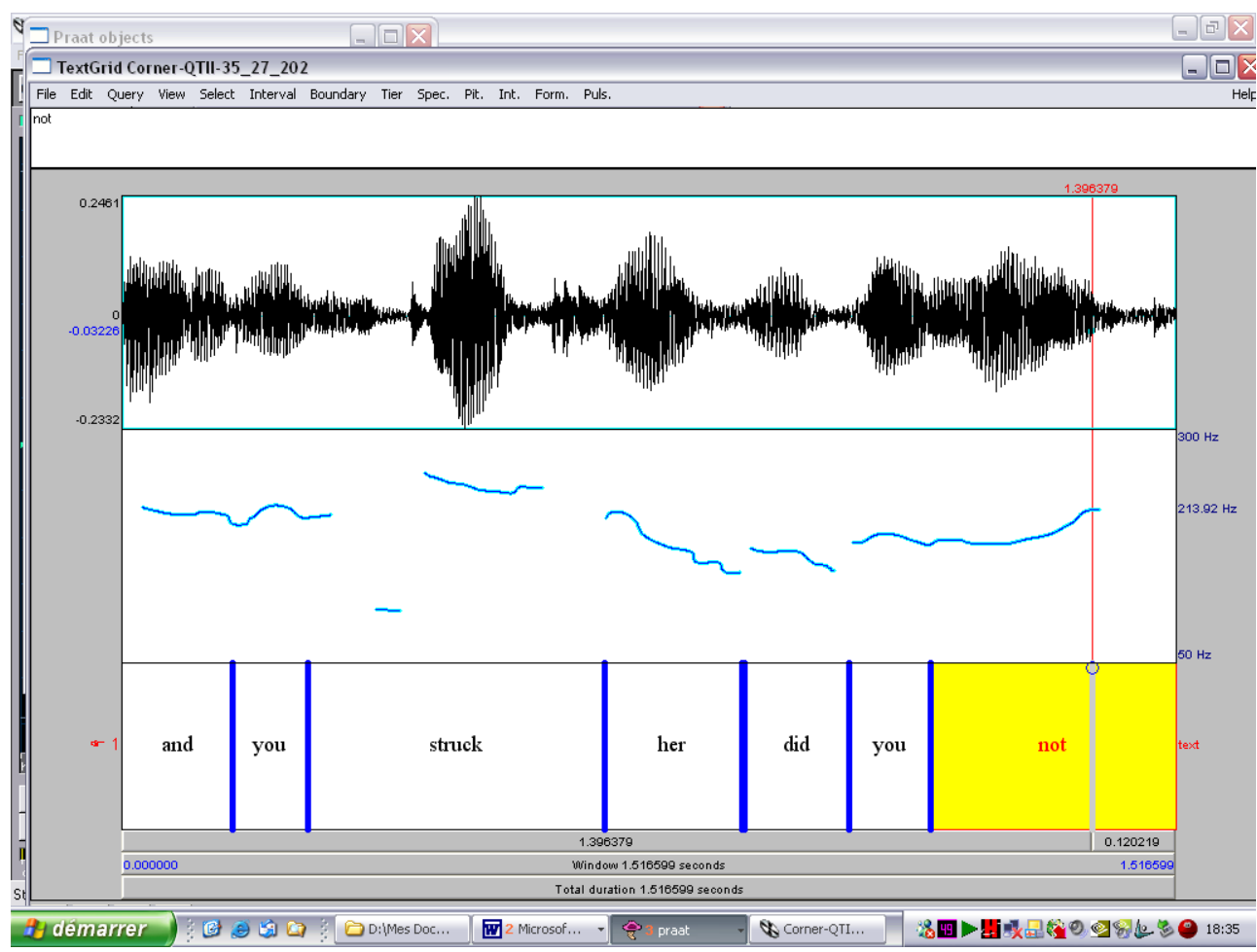

L'intonation légèrement montante sur le QTag dans l'interprétation qu'en propose l'actrice n'indique pas un quelconque doute de l'avocate sur le contenu de la base, qu'elle a au contraire asserté de manière emphatique grâce à l'emploi de la grande chute, mais est un appel explicite à McCullough afin qu'il prenne en charge la relation [I / strike her]. Ainsi, cette apparente prise en compte du co-énonciateur cache là encore une exigence de prise en charge, un forçage de consensus. Il n'est pas du tout question de donner la parole au co-énonciateur, mais bien de lui faire dire ce qu'on a envie d'entendre : face au juge, un aveu aurait bien plus de poids que la simple accusation portée. Cette stratégie échoue néanmoins, puisque l'accusé continue à nier et répond : No, I didn't hit her.

Or, si la petite montée sur le QTag n'est pas spécialement emphatique, le verbe de la base conjugue à la fois plage haute, durée et intensité, tous traits congruents par rapport à l'expression de l'exclamation. Quant au QTag, il présente un élément qui, sur le plan syntaxique, peut s'apparenter à une mise en relief: c'est not, la forme pleine de la négation, qui est employée, et qui constitue ici le noyau de l'unité intonative formée par le QTag. Dans les QTags, la présence de not est la marque d'un verrouillage de parcours : l'énonciateur tente explicitement d'éliminer la valeur inverse.

Si cet énoncé avec QTag n'est pas interrogatif, comme nous venons d'essayer de le montrer, il peut bien être analysé comme une exclamation, dont il présente les traits, sur le plan intonatif notamment. Le contexte s'y prête, puisqu'on a à nouveau un haut degré de différenciation entre les positionnements respectifs des co-énonciateurs. Examinons maintenant deux énoncés qui font suite au précédent exemple :

(4) Avocate de Fran Boyce: Plenty of people have arguments, you don't see them going to the police to have the other person arrested, do you?

(silence)

Avocate: Do you, Mr McCullough? 
McCullough : Could you repeat the question please?

Avocate : Why would Miss Boyce go to the trouble to report to the police that you assaulted her if you didn't?

McCullough: (I don't know)... she got mad... we had a fight. (CornerQTII-35.36.772)

(4a) Corner-QTII-35.36.772a :

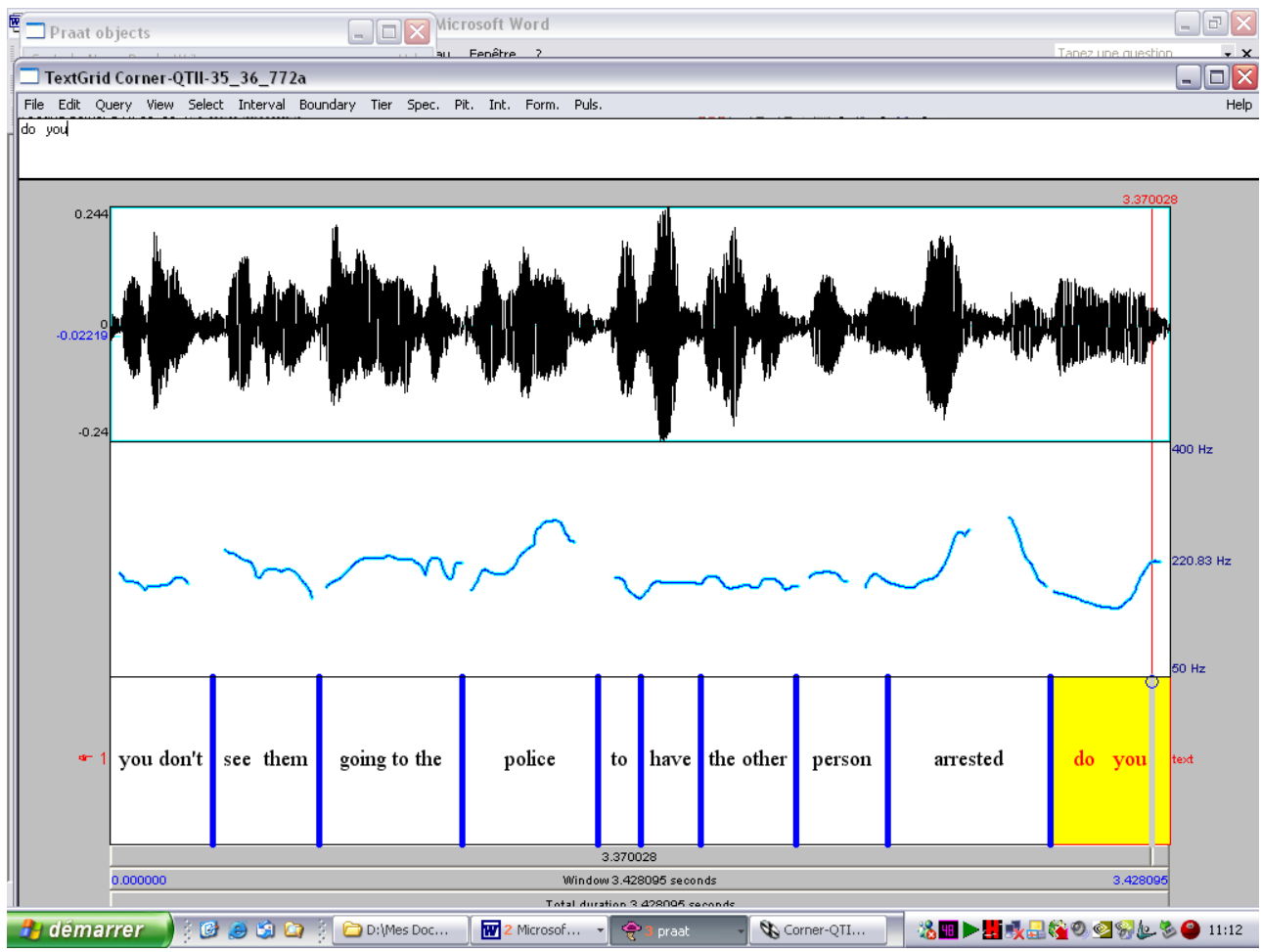

En ce qui concerne cet énoncé avec QTag, il n'est pas spécialement emphatique, ni exclamatif, si on en juge par l'amplitude intonative utilisée : on reste approximativement entre 200 et $250 \mathrm{~Hz}$, ce qui n'est pas très élevé pour une voix de femme, en particulier en ce qui concerne cette locutrice, comme on va le voir. Cet énoncé comporte un Rise-Fall sur le noyau de la base (arrested) suivi d'une petite montée sur le QTag. Il exprime en réalité la nécessité faible, comme le premier exemple que nous avons vu: il n'est donc pas exclamatif. A noter ici que le rôle de la petite montée sur le QTag est d'inciter le coénonciateur à réagir. Il ne s'agit pas d'une demande de confirmation dans le sens où l'avocate a déjà son avis sur le sujet ; elle veut simplement faire admettre par McCullough que sa ligne de défense n'est guère crédible. Or, celui-ci n'a aucune réaction. Au bout de quelques secondes de silence, l'avocate reprend d'une voix impérieuse : 


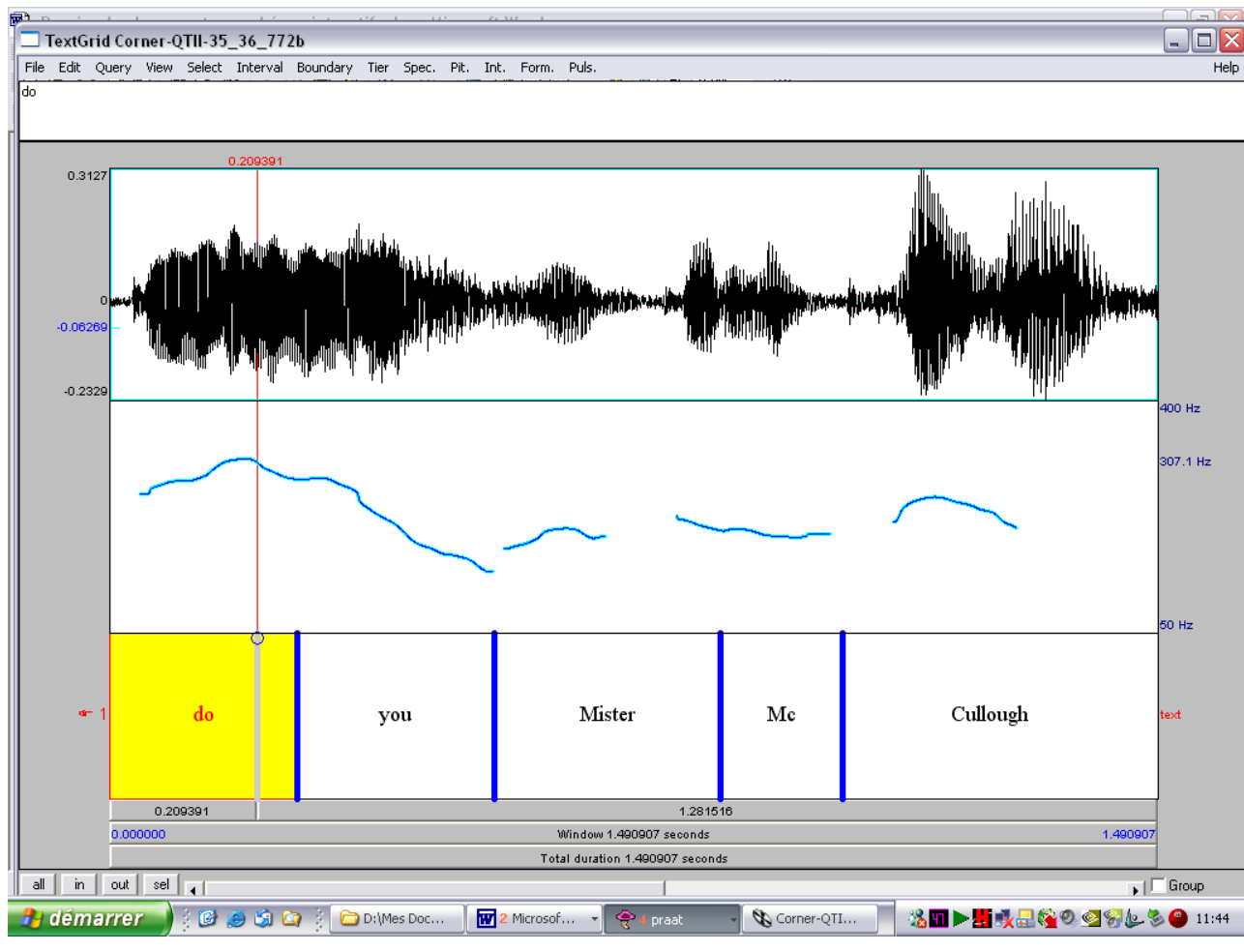

«Dites quelque chose, Mr McCullough!»

Il ne s'agit pas d'un énoncé avec QTag à proprement parler, car il n'y a pas de base. C'est la répétition $\mathrm{du} Q \mathrm{QTag}$ qui précède. On passe ainsi d'une petite montée, qui sollicitait une réaction de la part du co-énonciateur, à deux chutes, qui se révèlent encore plus impérieuses. Do you est réalisé au moyen d'une grande chute; c'est également le cas du terme d'adresse qui suit, sur lequel on note une intonation descendante de moindre amplitude sur la syllabe accentuée de Cullough, que l'on peut néanmoins qualifier de grande chute. L'emploi d'une intonation descendante montre que si le co-énonciateur est sollicité, ce n'est pas dans le but de lui laisser le choix de sa réponse, mais plutôt d'obtenir ce que l'on veut entendre de sa part., c'est-à-dire de confirmer le choix effectué au préalable par l'énonciateur.

En dépit de la possibilité de traduire en français par une exclamative (cf. supra), on est peut-être plus proche du domaine de l'injonction que de celui de l'exclamation néanmoins, le contexte polémique comme l'intonation employée soulignent la parenté d'une telle modalité avec l'exclamation, d'autant plus que le terme d'adresse est clairement destiné à interpeller et à rappeler à l'ordre le co-énonciateur (cf. Guillaume : 2005), tout comme dans l'exemple (2).

Intéressons-nous maintenant au cas des énoncés avec QTag qui présentent une polarité négative constante. Dans un tel cas de figure, la valeur négative mise en avant dans la base comme dans le QTag ne correspond pas au choix que l'énonciateur tente d'imposer au co-énonciateur, mais au positionnement affiché par le co-énonciateur, dont l'énonciateur se désolidarise, et avec lequel il exprime un fort désaccord, ce qui peut dès lors déclencher une interprétation exclamative. Le positionnement du co-énonciateur repris dans la base comme dans le QTag constitue un préconstruit (frayage), mais non 
contraire cette fois-ci. Ainsi, dans l'exemple suivant, Harry sait que sa femme le trompe ; dès lors, il reprend les paroles du co-énonciateur, mais sans pour autant partager son opinion:

(5) 'She's not a slut.'

I bite my lip. Did I just say that?

Harry is glaring at me now. 'Oh, she's not a slut, is she not?'

Nicole herself has no opinion on this fairly crucial subject.

'Even if she is a slut,' I respond, 'she's not your slut.' (Junkie 134)

« alors comme ça, ce n'est pas une trainée ! ( ? ${ }^{14}$ »

L'énoncé avec QTag n'est pas interrogatif ici, il permet simplement à l'énonciateur d'exprimer son désaccord avec ce qui vient d'être dit. Ceci relance du reste le débat, puisque le premier énonciateur corrige alors quelque peu ses propos : "Even if she is a slut, (...), she's not your slut.» A propos de cette catégorie d'énoncés (polarité négative constante), le linguiste australien W. McGregor note une propension à exprimer la désapprobation qui correspond bien à l'exemple ci-dessus :

“(..) the main context is strong disagreement with a proposition previously uttered by the addressee, or predictable from what they have said or done; at the same time, disapproval may be implied." (McGregor 1995b: 99 ; c'est nous qui mettons en gras)

Ainsi, les mélodies employées ${ }^{15}$ dans l'enregistrement sollicité (petite montée précédée d'une tête relative basse - ou take off, selon la terminologie d'o'Connor et Arnold (1973) sur la base, et petite montée sur le QTag) sont compatibles avec l'expression de la surprise comme de la désapprobation (cf. Nicaise et Gray 1998), tandis que l'on note une intensité très marquée et une assez grande amplitude intonative :

(5) Junkie 134- GF :

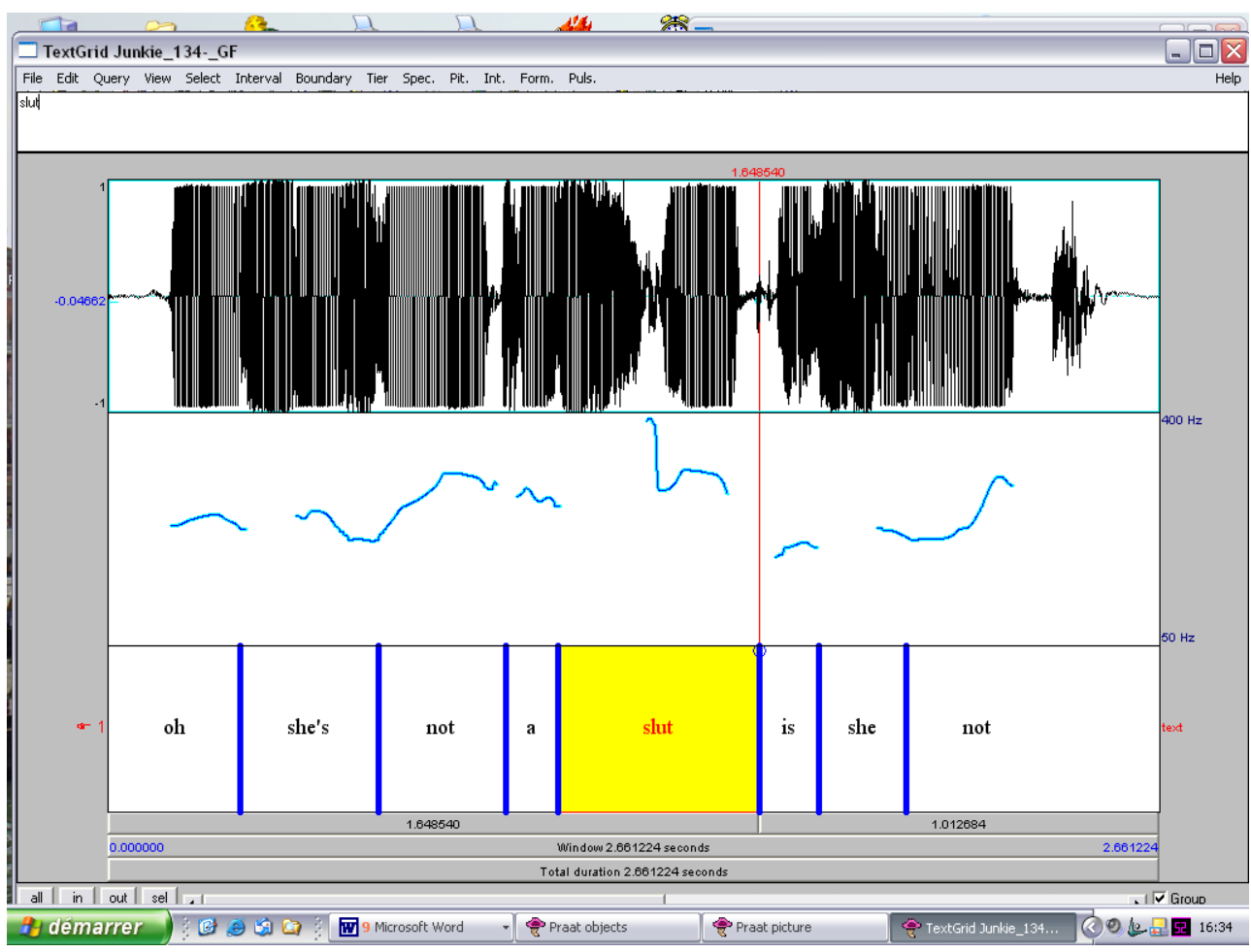


les exemples examinés jusqu'à présent mettent en scène des cas dans lesquels le contexte est celui d'un haut degré de différenciation entre les positionnements des coénonciateurs. Dans les énoncés exprimant la nécessité (exemples 2 à 4), l'énonciateur fait fi du préconstruit contraire dans l'espoir, souvent vain, d'effectuer un forçage de consensus. Dans l'exemple de polarité négative constante, la différenciation est au contraire reconnue par l'énonciateur, qui exprime néanmoins de la surprise, mais surtout du ressentiment, vis-à-vis du positionnement du co-énonciateur. Or, parmi les énoncés avec QTag, une autre catégorie encore nous semble pouvoir s'apparenter dans certains cas à l'expression de l'exclamation. Il s'agit d'énoncés dont la base ne comporte pas d'assertion, ce qui se marque, dans le cas qui nous intéresse ici, par la présence d'une inversion sujet / auxiliaire dès la base.

Contrairement aux exemples examinés jusqu'à présent, on ne retrouve pas dans le contexte de l'exemple (6) de différenciation entre les positionnements respectifs des coénonciateurs. Comme pour les énoncés comportant une polarité négative constante, on a dans le contexte un préconstruit non contraire, qui fournit le contenu de la base. Or, ce préconstruit semble tellement surprenant à l'énonciateur qu'il ne peut se résoudre à le prendre en charge :

(6) Are you going now are you? (McGregor 1995b : 100)

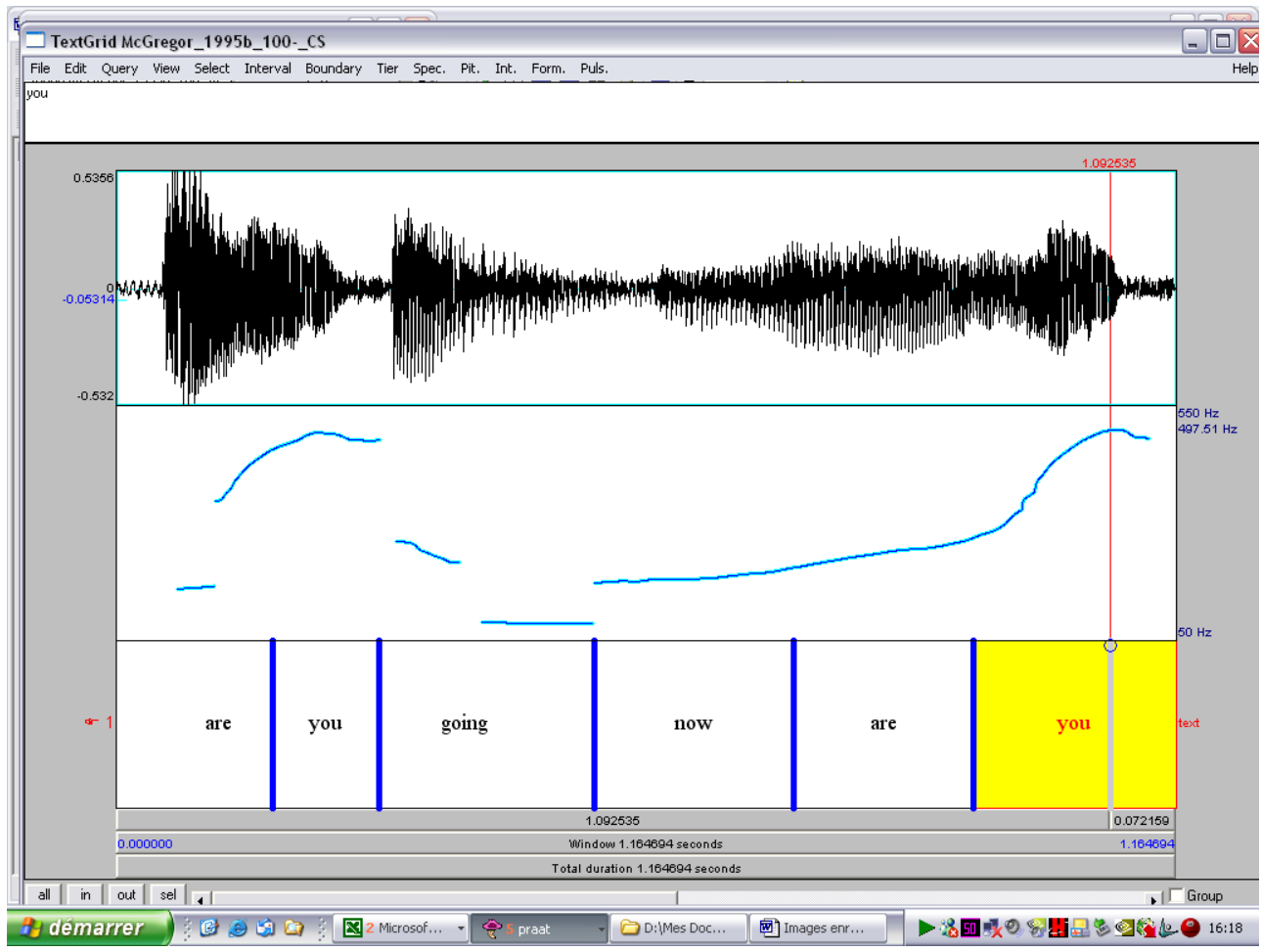

Concernant l'enregistrement sollicité de cet exemple, on note avant tout la très grande amplitude intonative employée par notre informatrice, entre 100 et $500 \mathrm{~Hz}$ environ, perceptible principalement au niveau de la grande montée sur le QTag. On est ici au-delà de la différence d'opinion, car l'action de la personne, qui se lève et rassemble ses affaires au beau milieu d'une importante réunion, semble tellement incongrue que l'énonciateur a du mal à y croire. Ceci explique la présence d'une inversion sujet / auxiliaire dès la base. 
On se retrouve dès lors proche de la définition classique de l'exclamation: les attentes de l'énonciateur sont prises en défaut. Néanmoins, le point commun avec les exemples qui précédaient est que la très grande surprise exprimée par l'énonciateur est tout de même directement liée à l'attitude du co-énonciateur : on n'a donc pas quitté la problématique de l'altérité.

En ce qui concerne une possible traduction en français, l'emploi d'un point d'exclamation ne serait pas incongru, mais la présence d'un point d'interrogation est tout de même plus vraisemblable dans ce cas précis :

"Alors comme ça, tu pars avant la fin?»

Pour conclure cette partie consacrée aux énoncés avec QTag pouvant recevoir une interprétation exclamative, examinons un dernier énoncé, qui appartient à la même catégorie que (6), mais qui se révèle en fait plus proche des exemples examinés précédemment dans la mesure où on est à nouveau en présence d'un conflit très net entre les positionnements respectifs des co-énonciateurs.

Charlotte est très en colère contre son fiancé, qui se montre particulièrement désinvolte à son égard. La base comporte une inversion sujet / auxiliaire, marque cardinale de nonassertivité, qui correspond non seulement à de la surprise, comme en (6), mais qui se double clairement ici d'une grande colère; on est dès lors proche des phénomènes observés en ce qui concerne la polarité négative constante en (5) :

(7) Charlotte: Do you know how lucky you are to have me? Do you know how we look? Do you know what people out there think when they see us together, do you?

Harry: Yeah, I know what people are thinking. I just didn't think you were one of them. (Sex and the City $6 ; 4$ )

Ainsi, l'absence d'assertion dans la base peut trahir un désaccord entre les coénonciateurs. Plutôt que d'assumer ce désaccord, l'énonciateur préfère interpeller le coénonciateur au moyen de la double inversion sujet / auxiliaire afin de faire pression sur ce dernier, qui se retrouve sommé de confirmer ou d'infirmer le préconstruit qui lui est attribué. L'emploi du QTag en (7) est de ce fait comme une perche tendue au coénonciateur afin qu'il montre des regrets pour ses actes. Néanmoins, cette stratégie se retourne ici contre Charlotte, puisque, en fin de compte, c'est Harry qui parvient à la culpabiliser.

Sur le plan de l'intonation, la hauteur extrême de la voix de l'actrice (entre 450 et $650 \mathrm{~Hz}$ en moyenne) traduit l'intensité de la colère du personnage. Le recours à des grandes montées sur la base comme sur le QT n'a rien d'interrogatif au sens où une question se poserait, il s'agit-là d'une manière d'interpeller vivement l'interlocuteur. 


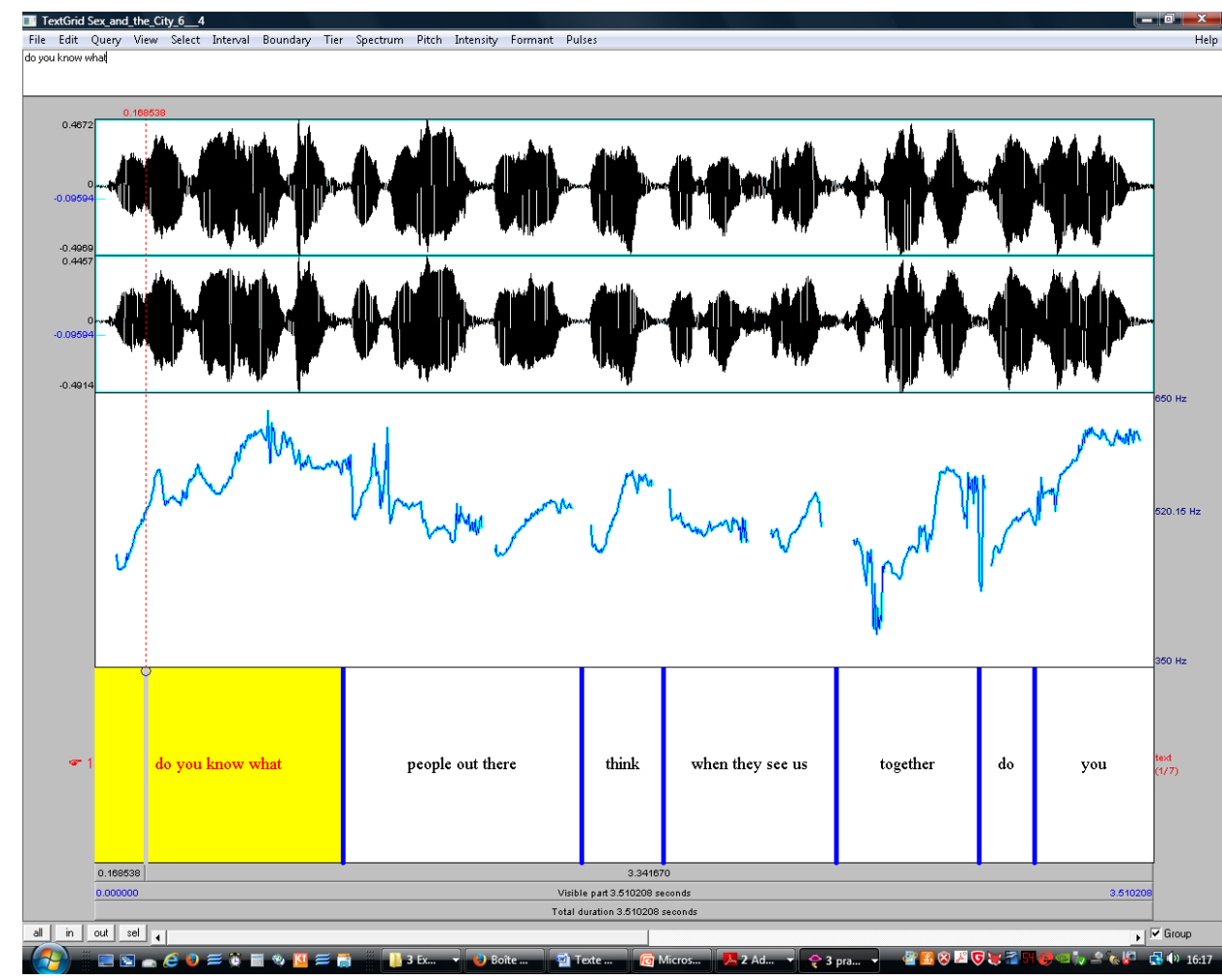

«Tu es conscient au moins de que ce les gens se disent en nous voyant ensemble?» ou:

«Tu réalises un peu... ?»

Quant aux marqueurs ("au moins », " un peu») auxquels nous avons recours dans la proposition de traduction, ils correspondent pour leur part, tout comme le QTag et les grandes montées de l'énoncé original, à une interpellation pleine de reproches du coénonciateur. On ne peut néanmoins pas aller jusqu'à employer un point d'exclamation.

Ainsi, les énoncés avec QTag pouvant être analysés comme des énoncés exclamatifs se rencontrent nécessairement dans un contexte polémique, dans lequel on note la plupart $\mathrm{du}$ temps une grande différenciation entre les positionnements respectifs des coénonciateurs. Celui-ci peut également correspondre à de la surprise. Si l'on ne trouve jamais de point d'exclamation après un QTag à l'écrit, on note en revanche des caractéristiques intonatives hautement compatibles avec un contexte exclamatif. Or, ceci est également le cas des énoncés comportant some que nous allons maintenant étudier.

\section{Les emplois exclamatifs de some}

Comme beaucoup de marqueurs exprimant une faible détermination, le quantifieur some est compatible avec de multiples valeurs. Parmi celles-ci, on trouve une valeur bien connue, parfois appelée valeur exclamative ou rhétorique. Cet emploi résolument qualitatif du marqueur est souvent mentionné dans la littérature spécialisée, mais rarement étudié en détail (à noter, néanmoins : Mazodier 1997, Gilbert 1999, Israel 1999, 
par exemple). Nous avons ainsi essayé de déterminer les principales caractéristiques de cet emploi de some :

- la représentation que l'on peut en tirer en termes de domaine notionnel,

- et surtout la façon dont la prosodie et la syntaxe participent à l'interprétation méliorative ou minorative de l'énoncé.

Cet emploi se retrouve d'ailleurs principalement à l'oral dans un contexte de discours entre plusieurs locuteurs et il révèle de façon privilégiée un travail d'ajustement et de régulation des représentations effectué en situation par rapport à un co-énonciateur.

\section{1/ Carastéristiques de some appréciatif}

\subsubsection{Valeur et représentation en termes de domaine notionnel}

Nous ferons tout d'abord quelques remarques théoriques sur la valeur de some dans ce type d'emplois. Some est fondamentalement la marque d'une instabilité, compatible avec différentes interprétations, et pouvant porter sur le paramètre QLT (qualitatif = opération de tri entre les occurrences par leurs propriétés) ou QNT (quantitatif = concerne tout ce qui est lié à la délimitation spatio-temporelle, au passage à l'existence d'une occurrence). Ici, l'indétermination de some, son « instabilité » est qualitative, puisque l'occurrence est valuée subjectivement. On retrouve donc un tri de propriétés, pour savoir si l'occurrence appartient (identification) ou non (différentiation) à un certain domaine notionnel. On sait que cette structuration d'un domaine notionnel s'effectue par rapport à un pôle de référence, qui peut prendre la forme soit d'un centre organisateur (occurrence typique qui possède les propriétés de la notion, ni plus, ni moins), soit d'un centre attracteur (représentation imaginaire, idéale, inaccessible, qui possède toutes les propriétés de la notion au plus haut degré possible).

Nous retrouvons ici la valeur de some qui nous paraît fondamentale, c'est-à-dire l'écart ou l'altérité par rapport au centre organisateur d'un domaine notionnel (hypothèse proposée par Mazodier 1997 : 124).

Dans cet emploi «exclamatif», l'indétermination de some permet de renvoyer soit au haut degré, c'est-à-dire au centre attracteur du domaine (interprétation méliorative, « un excellent $\mathrm{X} »)$, soit à la frontière, voire à l'extérieur du domaine considéré (interprétation minorative, « un piètre $X$ », " pas vraiment ce que j'appelle un $X$ », voire "pas un $X$ du tout pour moi »). Dans tous les cas, some peut s'interpréter comme un écart par rapport au centre organisateur, c'est-à-dire à la norme. L'occurrence introduite par some est donc « extra-ordinaire » (Mazodier 1997 : 124). Ce caractère qualitativement non quelconque est primordial et il peut expliquer que parfois l'interprétation ne soit ni méliorative, ni minorative, l'énonciateur indiquant simplement que l'occurrence n'est pas typique (étrange, surprenante, etc.). L'analyse proposée par E. Gilbert va dans le même sens :

«[Some indique que l'occurrence] fonctionne hors étalonnage, qu'elle est hors norme, et, plus précisément, dans ce cas où l'on part d'emblée de l'intérieur du domaine, qu'elle est en dehors des normes, et donc anormale. Une telle opération, qui marque une rupture par rapport au centre organisateur du domaine, peut orienter soit vers le complémentaire soit vers l'attracteur, point ultime du gradient représentant le plus haut degré imaginable de la notion, et donc aussi bien s'accommoder d'une lecture dépréciative que méliorative, voire, le cas échéant, laisser volontairement la place à une certaine ambiguïté ». (Gilbert 1999 : 17) 


\subsubsection{Reprise et ajustement inter-subjectif} jugement porté sur une occurrence déjà mentionnée dans le contexte avant. Dans ce cas, on a donc une reprise avec réajustement notionnel. On retrouve par exemple ce type d'ajustement inter-sujets dans un contexte de dialogue entre plusieurs locuteurs. L'énonciateur porte alors un jugement appréciatif sur ce que vient de dire le coénonciateur, comme en (8), (9) et (10):

(8) 'We were going to start a commune.'

Eleanor gave a short, sarcastic laugh. 'Some commune it would have been, with two writers in it.' (HT p. 32)

(9) 'Whatever you do, don't ask Sam about his height, said a friend. 'Or his toupée.' I didn't know till then that he wore a toupée. Some friend said Eleanor. (HT p. 7)

(10) 'As an assassin she fits the role. A woman who could throw acid in another woman's face is capable of anything. She's clever - up to a point - is Tina. She whisks from one place to another. Hence her nickname, The Butterfly'.

'Some butterfly'. (TS, p. 120). comme dans les exemples suivants. Il peut s'agir de reprises textuelles strictes (11 et 12), ou de reprises notionnelles (comme en 13 et en 14, <authority> étant une réélaboration notionnelle de $<$ know $>$ et $<$ bedside manner $>$ de $<$ be rough $>+<$ introduce oneself $>$ ).

(11) He invited me over to MIT to see some computer-generated art, and he kissed me in the elevator on the way out. It was some kiss! (T p. 208)

(12) Stanley: Lie Number One: All this squeamishness she puts on! You should just know the line she's been feeding to Mitch. He thought she had never been more than kissed by a fellow! But Sister Blanche is no ${ }^{18}$ lily! Ha-ha! Some lily she is! (SND p. 186)

(13) 'What the hell does she know about it?', he screamed. 'She never felt it, not once. Some authority she is! It's like listening to a plant describe the motives of a mammal!' (J. Irving, The World according to Garp, Emprunté à Dubos 1990, p. 183).

(14) My arm scar is about 8 to 10 inches, it sucks! I am sorry your surgeon was such an $A^{* *}$. I remember the day he came to see you in the hospital and I was there. He was so rough, and didn't even introduce himself - some bedside manner! (exemple proposé par Garrett Collins)

On peut également constater que la valeur méliorative ou minorative est souvent frayée dans le contexte avant. Ce phénomène apparaît bien dans l'exemple suivant, où l'on peut observer une accumulation d'adjectifs (tremendous, gigantic, huge, incredible, colossal, giant ...) qui marquent le caractère non quelconque de l'occurrence sur le plan QLT et frayent ainsi la valeur de haut degré.

(15) She had hardly spoken when there was a tremendous eruption in the lake. They heard a muffled thump. This was followed by a gigantic upheaval. A portion of the lake climbed vertically into the night. A huge billowing fountain which, by Tweed's estimate, far exceeded the famous sixty-foot high fountain of the shore of Geneva. It mounted higher and higher as tons of water were lifted to an incredible height. Then the elevated man-made fountain, like a colossal geyser, began to fall. They stood still as it generated 
giant waves which rolled majestically towards the shore and then crashed against the lake wall, hurling more water into the road.

'That was some bomb,' said Tweed. (TS, p. 380).

\section{dénombrement, de l'évaluation d'une quantité, mais d'une} globalement et soumise à la valuation subjective de l'énonciateur (comme avec un nom discontinu singulier). Après avoir souligné ces quelques caractéristiques des emplois exclamatifs de some, nous allons à présent tenter de voir comment la prosodie et la syntaxe participent à l'interprétation exclamative et à la construction du sens dans ces énoncés.

\subsection{Opérations énonciatives de l'oral et syntaxe}

\subsubsection{Rôle de la non réduction de la voyelle et de l'accentuation}

La première remarque que l'on peut faire concerne la qualité de la voyelle de some. Comme dans les emplois où la composante QLT est prépondérante ou au moins équipondérée, some prend sa forme pleine et se prononce donc [ $\mathrm{s} \wedge \mathrm{m}]$. L'opposition entre $/ \Lambda /$ et la forme réduite $/ \ni /$ constitue ainsi une première trace de la valeur de some dans nos énoncés "exclamatifs", mais il faut également prendre en compte d'autres phénomènes suprasegmentaux, tels que l'accentuation et l'intonation.

En effet, dans ses emplois appréciatifs, some n'a pas seulement sa voyelle pleine, il est aussi porteur d'un battement rythmique qui correspond également à un accent mélodique, comme nous le verrons dans la suite de cette étude. On voit bien là l'importance de la prise en considération des opérations énonciatives de l'oral, la nécessité de dépasser par exemple l'opposition entre mots lexicaux et mots grammaticaux (some faisant bien sûr partie de ces cas de "mots grammaticaux » pouvant être accentués), si l'on veut comprendre la construction du sens de ces énoncés. 

en outre remarqué que dans la transcription à l'écrit de ces formes, le point d'exclamation n'apparaissait pas systématiquement. Le lecteur n'a pas besoin de cette marque typographique pour reconnaître un énoncé exclamatif, puisque cette valeur passe essentiellement par des moyens prosodiques. Une chute haute sur le noyau, par exemple, peut suffire à introduire une valeur « exclamative » : 
(15) TS, P. 380 :

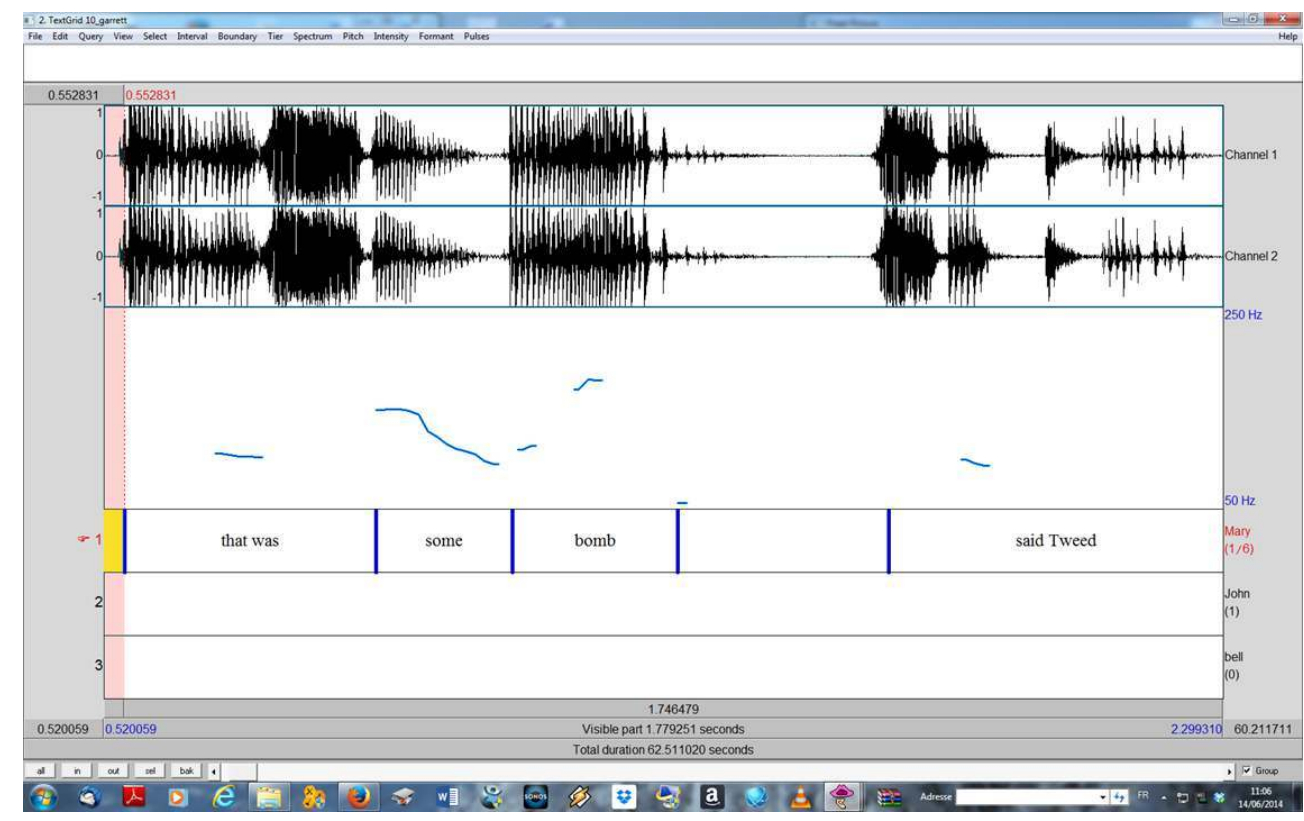

De plus, la forme étant a priori la même pour la valeur méliorative ou minorative (some + nom : aucun adjectif ou autre marque n'explicite cette valeur, même si le contexte lève souvent l'ambiguïté), l'intonation constitue une fois de plus un critère fondamental pour distinguer les différentes interprétations. Nous verrons enfin que la syntaxe joue aussi un rôle non négligeable, la thématisation de some et du nom qu'il détermine en tête d'énoncé étant caractéristique de l'interprétation minorative.

\section{a) Some appréciatif mélioratif}

Les exemples suivants figuraient sur le corpus que nous avons donné à lire à nos informateurs anglophones. Malgré quelques variations, les enregistrements que nous avons analysés révèlent des tendances relativement nettes au niveau du schéma intonatif et de la courbe mélodique :

(11) He invited me over to MIT to see some computer-generated art, and he kissed me in the elevator on the way out. It was 'some 'kiss! (T p. 208) (chute haute sur le second terme). 
(11) T p.208 :

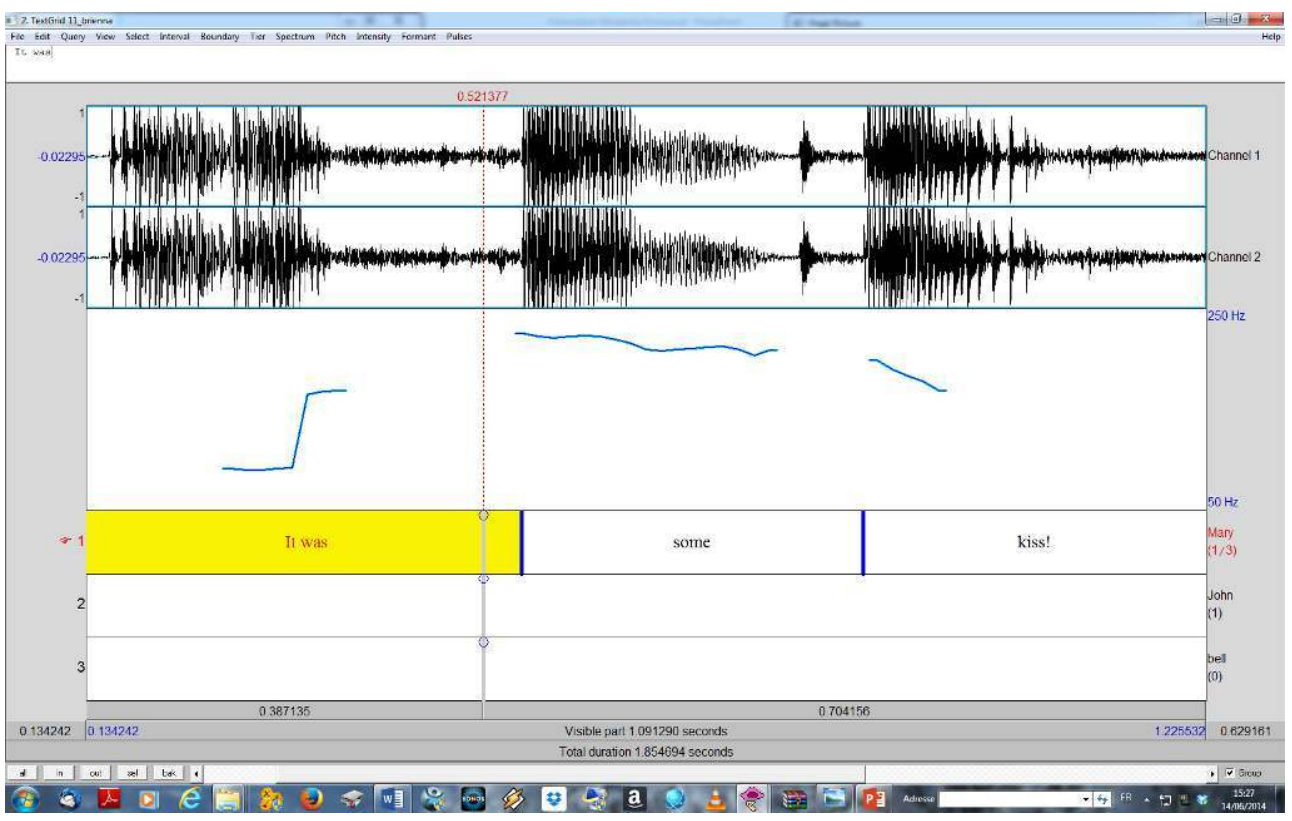

(15) She had hardly spoken when there was a tremendous eruption in the lake. They heard a muffled thump. This was followed by a gigantic upheaval. A portion of the lake climbed vertically into the night. A huge billowing fountain which, by Tweed's estimate, far exceeded the famous sixty-foot high fountain of the shore of Geneva. It mounted higher and higher as tons of water were lifted to an incredible height. Then the elevated man-made fountain, like a colossal geyser, began to fall. They stood still as it generated giant waves which rolled majestically towards the shore and then crashed against the lake wall, hurling more water into the road.

'That was 'some 'bomb,' said Tweed. (TS, p. 380). (19) The figure Persse had glimpsed earlier was indeed Angelica: she must have taken some detour, and had now reappeared on the path ahead of them, scarcely a hundred yards distant.

"That sure is 'some 'girl! She looks like a million dollars, has read everything you can name, and she can really run, can't she?". (SW, p. 44) 


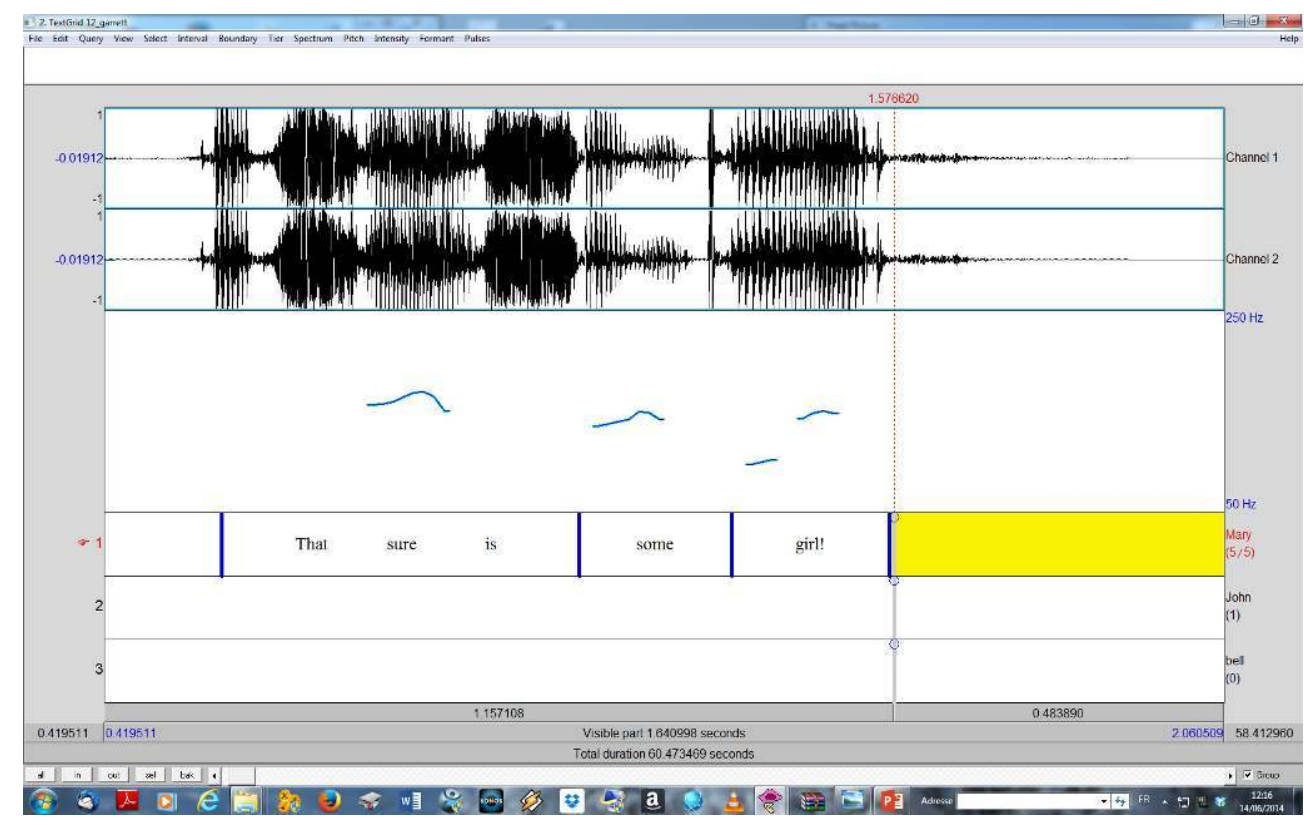

(20) The blonde was 'some 'dancer. She was one of the best dancers I ever danced with. (Mazodier 1997, p. 124)

(21) "After a time, Olla came back with it. I looked at the baby and drew a breath. Olla sat down at the table with the baby" [...] "Well", she said, "isn't that 'some 'baby!"

(Gilbert 1999, p. 17)

D'après les résultats obtenus dans notre corpus enregistré, la plupart des locuteurs anglophones que nous avons interrogés utilisent le même schéma intonatif pour ces énoncés. On remarque ainsi un contour particulier, avec une forte chute intonative (high fall), une amplitude importante qui signale que l'énoncé est plus qu'une simple assertion prise en charge par l'énonciateur, et participe donc à la construction de la valeur exclamative appréciative.

Du point de vue de la syntaxe et de l'agencement de ces énoncés, on remarque qu'il n'y a pas de thématisation du groupe nominal introduit par some et que la copule be à valeur d'identification apparaît de façon quasi-systématique. On a donc très souvent le schéma : it / that be + some $\mathrm{X}$. La présence de be en position canonique semble donc bloquer la valeur minorative. Pour exprimer celle-ci, on retrouve beaucoup plus d'énoncés sans be ( some $\mathrm{X}$ ) ou avec une antéposition du groupe nominal introduit par some (some $\mathrm{X}+$ sujet + be). On a donc une interaction entre la prosodie et la syntaxe pour donner sa valeur référentielle à une forme indéterminée et potentiellement ambiguë.

\section{b) Some appréciatif minoratif :}

Lorsque some prend une valeur minorative (renvoi à la frontière ou à l'extérieur du domaine notionnel), nous avons remarqué que le schéma intonatif était relativement différent. Les énoncés de notre corpus qui correspondent à ce cas de figure sont les suivants : 
(8) 'We were going to start a commune.'

Eleanor gave a short, sarcastic laugh. 'Some commune it would have been, with two writers in it.' (HT p. 32)

(9) 'Whatever you do, don't ask Sam about his height, said a friend. 'Or his toupée.' I didn't know till then that he wore a toupée. Some friend! Said Eleanor. (HT p. 7)

(10) 'As an assassin she fits the role. A woman who could throw acid in another woman's face is capable of anything. She's clever - up to a point - is Tina. She whisks from one place to another. Hence her nickname, The Butterfly'

'Some butterfly'. (TS, p. 120).

(12) Stanley: Lie Number One: All this squeamishness she puts on! You should just know the line she's been feeding to Mitch. He thought she had never been more than kissed by a fellow! But Sister Blanche is no lily! Ha-ha! Some lily she is! (SND p. 186)

(13) 'What the hell does she know about it?', he screamed. 'She never felt it, not once. Some authority she is! It's like listening to a plant describe the motives of a mammal!' (J. Irving, The World according to Garp).

(14) My arm scar is about 8 to 10 inches, it sucks! I am sorry your surgeon was such an $A^{* *}$. I remember the day he came to see you in the hospital and I was there. He was so rough, and didn't even introduce himself - some bedside manner! (exemple proposé par Garrett Collins)

(16) [Blanche is singing in the bathroom a saccharine popular ballad which is used contrapuntally with Stanley's speech.

Stanley]: Some canary-bird, huh! (SND p. 186)

second one of the war, and its approach revives ironic memories of a confident phrase bandied about in August 1914. "It'll all be over by Christmas" - remember that?' Burgess says to Minnie and Joan as they sit round the kitchen table. 'Some 'opes!' (AA, p. 25)

(22)'At the request of Mr Gatsby we are going to play for you Mr Vladmir Tostoff's latest work, which attracted so much attention at Carnegie Hall last May. If you read the papers you know there was a big sensation.' He smiled with jovial condescension, and added: 'Some sensation!' Whereupon everybody laughed. (GG, p. 51)

Contrairement aux énoncés comportant un some mélioratif que nous avons vus précédemment, on a ici un ton beaucoup plus égal, avec une chute intonative de plus faible amplitude et une amorce relativement grave (sur some). Le schéma intonatif est donc différent de celui des "vraies exclamatives », d'où l'effet ironique obtenu («tu parles d'un X!, un drôle de X ! »). En employant ce schéma intonatif, l'énonciateur signale ainsi que l'occurrence n'a pas vraiment, voire pas du tout, les propriétés notionnelles de la classe en question. Il n'est d'ailleurs pas inintéressant de noter comment ces exemples ont été traduits en français : en (8), « elle était bien partie, la communauté, avec deux écrivains!» (Lodge, D. 2002. Les Quatre Vérités, traduit par Suzanne Mayoux, Editions Rivages), en (9), «tu parles d'un ami !» (Lodge, D. 2002. Les Quatre Vérités, traduit par Suzanne Mayoux, Editions Rivages), en (17), « comptez-y!», (Lodge, D. 2002. L'Auteur ! L'auteur ! traduit par Suzanne Mayoux, Editions Rivages), et en (22), « pour du bruit, elle a fait du bruit!» (Fitzgerald, FS. 1925, traduction de Michel Viel, 1991, Lausanne : L'Âge d'homme).

Du point de vue de l'agencement syntaxique, ces énoncés se démarquent aussi des some mélioratifs de façon significative et nous retrouvons un type de prédication différent. L'observation la plus marquante que nous pouvons faire est que, dans ces exemples, some 
et le nom qu'il détermine apparaissent systématiquement en tête d'énoncé. Très souvent, ils peuvent apparaître seuls, dans ce que l'on pourrait appeler un énoncé averbal, du type «some $\mathrm{X}$ !». Cette thématisation du groupe nominal introduit par some crée un lien explicite avec le contexte avant, et, par là même, la réfutation ironique (déstabilisation d'une relation préconstruite) est d'autant plus apparente. Lorsque le marqueur d'identification be apparaît dans l'énoncé, some $+\mathrm{N}$ reste en position initiale et l'on a donc: some $+\mathrm{N}+\mathrm{C}_{0}$ (sujet syntaxique) + be, comme en (12) et en (13):

(12) Stanley: Lie Number One: All this squeamishness she puts on! You should just know the line she's been feeding to Mitch. He thought she had never been more than kissed by a fellow! But Sister Blanche is no lily! Ha-ha! Some lily `she is! (SND p. 186)

(13) 'What the hell does she know about it?', he screamed. 'She never felt it, not once. Some authority 'she is! It's like listening to a plant describe the motives of a mammal!' (J. Irving, The World according to Garp).

Dans ce contexte, on voit bien que la configuration syntaxique est contraignante et l'on ne pourrait absolument pas avoir : *she is some lily!, *she is some authority! avec la même valeur.

Nous avons également remarqué que, pour ces exemples, la plupart des informateurs anglophones consultés placent un accent contrastif sur le pronom personnel, qui marque sans doute le caractère incongru de la relation préconstruite entre le sujet et la propriété attribuée (par exemple : <she - be never more than kissed by a fellow> en 12 et <she - know> en 13). L'opération que reflète cet accent contrastif, c'est bien le choix d'un sujet en particulier (ouverture d'un paradigme et choix d'un élément à l'intérieur de celui-ci) par rapport à la propriété considérée. On a par conséquent ici la mise en place et la prise en compte d'une altérité.

Enfin, pour les énoncés dont l'interprétation n'est ni clairement méliorative, ni clairement minorative, comme en (17), il nous semble que le schéma intonatif est légèrement différent des deux autres cas de figure analysés précédemment, mais nous n'avons malheureusement pas recueilli assez d'exemples pour pouvoir être vraiment certain de ces tendances :

(23) Fernandez looked around the room with a proprietary air, then shook his head in disgust. 'You've got some place here, my friend. If you don't mind me saying so, it reminds me of a coffin. One of those pine boxes they bury bums in.' (Gilbert 1999, p. 17)

61 Il nous semble néanmoins que l'ambiguïté de ces énoncés se retrouve dans l'intonation, qui n'a pas vraiment les traits caractéristiques des autres cas étudiés. Les enregistrements obtenus ne sont pas totalement homogènes, mais deux éléments paraissent ressortir :

- la chute intonative n'a pas une très grande amplitude (contrairement aux énoncés mélioratifs)

- on ne retrouve pas l'attaque dans les graves (à l'inverse des réfutations ironiques).

Ceci confirmerait bien que l'intonation, en combinaison avec d'autres composantes, joue un rôle crucial pour déterminer les différentes valeurs possibles du some appréciatif dans son emploi exclamatif. 


\section{Conclusion}

62 employés ne sont pas toujours typiques de l'exclamation, l'intonation, en revanche, tend à montrer une proximité réelle avec les énoncés exclamatifs, notamment au travers de l'amplitude employée, de l'intensité ressentie et de la longueur des voyelles.

Néanmoins, si les exemples comportant some appréciatif se révèlent à l'analyse des énoncés exclamatifs à part entière, ce qui est corroboré par le fait que beaucoup d'entre eux sont ponctués par un point d'exclamation, le cas des énoncés avec QTag reste certainement plus nuancé. Dans ce cas précis, il pourrait peut-être s'avérer plus judicieux de parler d'énoncés pseudo-exclamatifs, qui n'auraient pas toutes les caractéristiques des énoncés exclamatifs. On serait dès lors confronté à un effet de sens exclamatif plutôt qu'à un énoncé exclamatif proprement dit.

deuxième point essentiel que nous avons tenté de démontrer est l'importance de ne pas dissocier les différentes composantes du langage. Nous avons en effet remarqué une interaction constante entre la prosodie, ce que l'on pourrait appeler les opérations énonciatives de l'oral (sons, accentuation, rythme, intonation) et la syntaxe. Tous ces éléments apportent en effet leur contribution à la construction du sens et permettent parfois de construire des interprétations très différentes avec les mêmes formes. Dans le cas des emplois appréciatifs de some, nous avons observé que les différentes valeurs appréciatives possibles résultaient principalement d'une combinaison intonation / syntaxe avec des configurations différentes pour chacun des cas analysés. En prolongement de ce travail, on pourrait envisager de comparer les résultats obtenus ici avec d'autres énoncés exclamatifs, afin d'approfondir l'étude, très vaste et encore largement inexplorée, de la « grammaire orale » de l'anglais.

\section{BIBLIOGRAPHIE}

Culioli, Antoine. 1974. « A propos des énoncés exclamatifs. » Langue française. 22 : 6-15. http:// www.persee.fr/web/revues/home/prescript/article/lfr_0023-8368_1974_num_22_1_5671 Culioli, Antoine. 1999. Pour une linguistique de l'énonciation. Domaine notionnel. Tome III. Paris : Ophrys.

Danon-Boileau, Laurent et Morel Mary-Annick. 1995. Faits de langues 6 : L'Exclamation. http:// www.persee.fr/web/revues/home/prescript/article/flang_1244-5460_1995_num_3_6_999

Filippi-Deswelle, Catherine, éd. 2012 L'ajustement dans la TOE d'Antoine Culioli. Collection linguistique Épilogos, 3. Rouen : Publications Électroniques de l'ERIAC. http://eriac.net/ category/publications/publications-electroniques/epilogos/l-ajustement-dans-la-toe-d-antoineculioli/

Gaudy-Campbell, Isabelle. 2000. « Le question-tag descendant : marque de questionnement ou d'égocentrage?» Anglophonia / Sigma. 8 : 167- 80.

Corela, $12-2$ | 2014 
Gaudy-Campbell, Isabelle. 2002. « Le Question-tag descendant : juxtaposition de deux unités?» Construire et reconstruire en linguistique anglaise. Syntaxe et sémantique. Travaux du CIEREC 107. 169-183. Saint-Etienne : PU de Saint-Etienne.

Gilbert, Eric. 1999. «Some et la construction d'une occurrence », in Détermination nominale et individuation. Ed. J.-C. Souesme. Cycnos. 16.

Groussier Marie-Line. 1995. « L'exclamatif, l'intensif et le focalisé. » Faits de langues 6 : 217-229. http://www.persee.fr/web/revues/home/prescript/article/

flang_1244-5460_1995_num_3_6_1024

Groussier, Marie-Line et Riviere, Claude. 1996. Les mots de la linguistique. Lexique de linguistique énonciative. Paris : Ophrys.

Guillaume, Bénédicte. 2005. « Terme d'adresse et question tag en co-occurrence : redondance ou stratégie énonciative? » Modèles linguistiques. Nomination, noms propres, termes d'adresse II. Tome XXVI, 2. Vol. 52 : 113-134.

Guillaume, Bénédicte. 2006. Approche énonciative des questions tags en anglais contemporain. Numéro spécial des Cahiers de Recherche. Paris : Ophrys.

Huart, Ruth. 2002. Grammaire orale de l'anglais. Paris : Ophrys.

Israel, Michael. 1999. « Some and the pragmatics of indefinite construal », Proceedings of the Berkeley Linguistics Society $25: 169-182$

Kerfelec, Valérie. 2009. L'exclamation en français et en anglais. Formes, sens, effets. Collection Langue \& Langage. Aix-en-Provence : Publications de l'Université de Provence.

Mazodier, Catherine. 1997. " "I must have read it in some article" : instabilité qualitative de some + discontinu singulier. » La composante qualitative : déterminants et anaphoriques. Cahiers de recherche Tome 7. A. Deschamps et J. Bouscaren, éds. Paris : Ophrys.

McGregor, William. 1995a. «Ja hear that didja?: Interrogative Tags in Australian English. » Te Reo. $38: 3-35$.

McGregor, William. 1995b. « The English ‘Tag Question': A New Analysis, is(n't) it? » On Subject and Theme : A Discourse Functional Perspective. Eds R. Hasan et P.H. Fries : 91-121. Amsterdam : John Benjamins.

MOREL, Mary-Annick. 1995. «L'intonation exclamative dans l'oral spontané. » Faits de langues 6 : 63-70. Doi : 10.3406/flang.1995.1006. http://www.persee.fr/web/revues/home/prescript/article/ flang_1244-5460_1995_num_3_6_1006

Morel, Mary-Annick et Danon-Boileau, Laurent. 1998. Grammaire de l'intonation. L'exemple du français. Paris : Ophrys.

Martin, R. 1987. « La sémantique de la phrase exclamative. » Revue d'Études Slaves. LDC/3.

Nicaise, Alain et Gray, Mark. 1998. L'intonation de l'anglais. Paris : Nathan Université.

O'Connor, J.D. et Arnold, G.F. 1973. Intonation of Colloquial English. 2ème édition. Londres : Longman.

Paulin Catherine. 2007. La Fonction expressive. Volume 1. Besançon : Presses Universitaires de Franche-Comté.

Rett, Jessica. 2011. 'Exclamatives, degrees and speech acts.' Linguistics and Philosophy. 34:411-442. 


\section{ANNEXES}

Corpus QTags.

GALLAGHER, Brian. 2000. The Feng Shui Junkie. Londres : Orion.

Abréviation Junkie

McGREGOR, William. 1995b. « The English ‘Tag Question': A New Analysis, is(n't) it? » On Subject and Theme: A Discourse Functional Perspective. Eds R. Hasan et P.H. Fries : 91-121. Amsterdam : John Benjamins.

Abr. McGregor 1995b

RENDELL, Ruth. [1989] 1990. The Bridesmaid. Londres : Arrow.

Abr. Bridesmaid

Sex and the City. 2003. « Pick-a-Little, Talk-a-Little. » Saison 6, épisode 4. Créé par D. Starr. HBO - Time Warner Entertainment Company. Avec K. Davis.

Abr. Sex and the City

The Corner. 2000. Réalisé par Charles Dutton. D’après le livre de D. Simon et E. Burns. HBO Miniseries.

Abr. Corner

The Sculptress. 1996. Réalisé par Stuart Orme. D’après le roman de M. Walters. Red Rooster Film and Television Entertainment for the BBC. Avec P. Quirke et C. Goodall.

Abr. Sculptress

Corpus some.

ELLIS, R. (2000) Framed. Londres : Headline Book Publishing.

Abréviation F.

FITZGERALD, F. S. (1926) The Great Gatsby. Londres : Penguin, 1990.

Abr. GG.

FORBES, C. (1998) The Sisterhood. Londres : BCA.

Abr. TS.

IRVING, J. (1978) The World according to Garp.

LODGE, D. (1984) Small World. Londres : Penguin, 1985.

Abr. SW.

---. (1999) Home Truths. Londres : Penguin.

Abr. HT.

---. (2001) Thinks. Londres : Secker \& Warburg.

Abr. T.

---. (2004) Author, Author. Londres : Secker \& Warburg. 
Abr. AA.

MILLER, A. (1949) Death of a Salesman. Londres : Penguin Classics, 2000.

Abr. DS.

ROTH, P. (1969) Portnoy's Complaint. Londres : Vintage edition, 1995.

Abr. PC.

WILLIAMS, T. (1947) A Streetcar Named Desire. Londres : Penguin, 1962.

Abr. SND.

\section{NOTES}

1. Par exemple, L. Danon-Boileau et M.-A. Morel décrivent l'exclamation comme une " mise en défaut des anticipations du locuteur, ou plutôt une tension contradictoire entre "ce qui est et ce qu'on pouvait penser qui serait" " (1995 : 9, citant Martin 1987). Ceci peut être rapproché de la définition donnée par J. Rett : "the utterance of an exclamation expresses a violation of the speaker's expectation » $(2011: 412)$.

2. Au même titre, par exemple, que le temps, la personne, le nombre...

3. Ainsi, how beautiful it is! peut être considéré comme une version hyper assertée de it's beautiful. Comme l'explique A. Culioli : «Il s'agit bien d'assertives, mais avec un quelque chose en plus qui se marque par des procédés divers, lexicaux, prosodiques, syntaxiques » (1974:6).

4. A ce sujet, nous renvoyons aux interrogations de C. Paulin (2007:14), qui envisage notamment de penser la fonction expressive en termes de surimposition.

5. Si la prise en compte des marqueurs prosodiques semble devoir primer sur celle des marqueurs du niveau graphique, telle la ponctuation, dans la mesure où ces derniers ne représentent qu'une perception subjective du niveau oral, nous nous intéressons néanmoins à l'ensemble des signes pouvant être rapportés à l'expression de l'exclamation. En effet, notre corpus est constitué aussi bien d'exemples écrits (dont certains ont été produits oralement par des locuteurs natifs à notre demande) que d'exemples oraux (extraits de séries télévisées, et c'est alors nous qui les avons transcrits pour les besoins du présent travail).

6. En effet, sur le plan prosodique, l'exclamation se marque très régulièrement par une « amplification des paramètres intonatifs (intensité, durée, hauteur) » (Morel 1995 : 63) ainsi que par le recours à une "plage intonative haute (plus haute que le niveau habituel du locuteur" (Morel 1995 : 64). A ce sujet, voir aussi Groussier 1995, Nicaise et Gray 1998 et Huart 2002.

7. A la suite d'I. Gaudy-Campbell, nous nommons «base » le segment d'énoncé qui précède le QTag proprement dit. Ainsi, dans it's nice, isn't it?, la base correspond à it's nice tandis que isn't it? est le QTag.

8. En ce qui concerne les captures d'écran tirées du logiciel Praat (créé par P. Boersma et D. Weenink de l'Université d'Amsterdam), elles ont été obtenues soit à partir de la bande-son de séries télévisées britanniques ou américaines, soit en sollicitant des informateurs anglophones de naissance de diverses origines, à qui l'on demande de lire l'énoncé au sein de son contexte le plus large possible, et sans lui préciser sur quel élément en particulier porte notre étude. Concernant la lecture de ces captures d'écran, précisons que la ligne bleue médiane correspond aux variations de la hauteur de la voix (calculées en Herz), tandis que le spectrogramme restitue l'intensité. Nous nous efforçons par ailleurs de faire coïncider la transcription graphique du bas avec les phénomènes prosodiques des deux autres lignes. 
9. Nous proposons, à titre indicatif, des traductions de certains énoncés, qui sont assez libres dans la mesure où nous considérons que le phénomène des QTags n'a pas d'équivalent direct en français.

10. Nous empruntons cette expression à M.-A. Morel et L. Danon-Boileau (1998) ainsi qu'à I. Gaudy (2000).

11. "L'opération de " parcours " consiste à parcourir toutes les valeurs assignables à l'intérieur d'un domaine sans pouvoir s'arrêter à une valeur distinguée » (Culioli 1999: 48). Il faut noter néanmoins que, dans un QTag, le parcours entre deux valeurs est forcément tronqué dans la mesure où la présence du choix d'une valeur dans la base, lorsque celle-ci est assertée, correspond à un frayage. Ainsi, en réalité, une des deux valeurs est largement privilégiée par l'énonciateur : elle est présentée comme étant la bonne valeur (sauf cas particuliers, par exemple celui des bases de forme interrogative : nous en verrons deux exemples plus loin).

12. Voir également Groussier 1995 : 222 pour une analyse un peu différente, bien qu'elle ait lieu dans le même cadre théorique, de la compatibilité entre inversion sujet / auxiliaire et expression de l'exclamation.

13. A noter que le contexte d'un tribunal est particulièrement propice à des énoncés avec QTags exprimant la nécessité forte, et donc compatibles avec l'expression de l'exclamation. En effet, un avocat procédant à l'interrogatoire d'un accusé ou bien d'un témoin tient le rôle d'un rhéteur ; il n'est pas en quête de vérité, mais cherche à démontrer la version des faits la plus favorable à son client. D'où le recours à une intonation souvent emphatique (plage haute, intensité, longueur, grande chute...), en co-occurrence avec un QTag, dont le rôle est de donner l'impression que l'on invite le témoin à s'exprimer, alors qu'en réalité on ne cherche qu'à le manipuler.

14. Pour la traduction française, il est permis ici d'hésiter entre point d'exclamation et point d'interrogation, qui pourraient tous deux convenir à notre avis.

15. Il ne faut pas tenir compte du trait vertical au-dessus du $s$ de slut.

16. L'absence de ponctuation dans la transcription de l'exemple relevé par W. McGregor peut faire hésiter quant au statut de now. En revanche, le contexte qu'il donne (une personne s'en allant avant la fin) rend évident le fait que now est complément circonstanciel de temps. Or, on dirait que notre informatrice l'a plutôt interprété comme faisant partie du QTag. Par ailleurs, le trait bas qui semble représenter ing est vraisemblablement une erreur de transcription commise par Praat; en effet, la voix de CS ne peut pas descendre si bas, pour des raisons physiologiques.

17. La pseudo chute sur la dernière syllabe de together ne correspond pas au son de la voix.

18. On voit bien apparaître ici la parenté entre no et some à valeur qualitative, no renvoyant dans ce cas directement à l'extérieur du domaine notionnel, alors qu'avec some on peut rester à la frontière du domaine en question.

\section{RÉSUMÉS}

Ce travail, inscrit dans le cadre de la Théorie des Opérations Enonciatives d'Antoine Culioli, s'intéresse à certains emplois des question tags, ainsi que du quantifieur some en contexte exclamatif. Nous voulons ici montrer que ces marqueurs, bien que non spécialisés dans ce domaine, peuvent exprimer des effets de sens exclamatifs, car ils présentent des caractéristiques sémantiques, syntaxiques et prosodiques compatibles avec la construction de l'exclamation. Notre étude se fonde sur un corpus d'exemples authentiques provenant de sources variées 
(British National Corpus, romans et séries télévisées) d'anglais britannique et américain. Au travers de l'étude du corpus, tant sous sa forme écrite que sous sa forme orale (enregistrée ou sollicitée), il apparaît que l'interprétation exclamative que peuvent recevoir ces énoncés tient essentiellement à un contexte polémique. C'est donc le haut degré (au sens culiolien du terme) d'altérité rencontré dans les positionnements respectifs des co-énonciateurs qui facilite, voire déclenche, une interprétation exclamative. Au niveau intonatif, le contexte exclamatif se trouve matérialisé par une intensité caractéristique (hauteur et amplitude de la voix, allongement des voyelles...), comme le montre l'analyse intonative réalisée au moyen du logiciel Praat.

In this paper we will discuss the exclamative values of some question tags and of the quantifier some, within the framework of A. Culioli's Theory of Enunciative Operations. Although these markers are not typical of the expression of exclamation, we aim at showing that they are compatible with an exclamative value, because they present certain semantic, syntactic and prosodic features which are also found in exclamatory sentences. Our study is based on a corpus of attested examples of British or American English sampled from various sources (namely, the British National Corpus as well as novels and TV series), both in their written and in their oral forms. The main conclusion to be drawn from the analysis of the context of the examples is that the exclamative interpretation which can be felt is linked to a strong disagreement between the speakers. It is therefore a high degree (which is a technical term in Culioli's framework) of controversy which tends to generate the exclamative interpretation of such phrases, which are not, otherwise, typical of exclamatory sentences. At the level of intonation, the recourse to the Praat software helps to evidence such phenomena as pitch variation or vowel lengthening, which for their part are essential as far as an exclamative interpretation is concerned.

\section{INDEX}

Mots-clés : exclamation, question tag, some, altérité, intonation

Keywords : exclamative sentence, controversy

\section{AUTEURS}

\section{BÉNÉDICTE GUILLAUME}

Université de Nice Sophia Antipolis, CNRS, BCL, UMR 7320

\section{EMMANUEL BAUMER}

Université Paris-Diderot, Sorbonne Paris Cité, CLILLAC-ARP EA 3967 